\title{
Optic glioma of childhood
}

\section{Natural history and rationale for conservative management}

\author{
WILLIAM F. HOYT AND SAHAG A. BAGHDASSARIAN* \\ Departments of Ophthalmology, Neurology, and Neurological Surgery, University of California \\ Medical Center, San Francisco
}

Controversy surrounds the management of childhood optic gliomata in the anterior visual pathways. This stems from insufficient knowledge of their natural history, varying definitions of their cytology, preoccupation with surgical indications for their excision, and lack of agreement regarding the role of radiation therapy. Although long survivals have frequently been reported, optic gliomata continue to be treated by surgical measures that often increase their morbidity.

The first requisite for rational management of any slow-growing tumour is knowledge of its natural history. This report documents the long-term clinical behaviour of optic gliomata seen in $3^{6}$ patients at the University of California Medical Center in San Francisco, and formulates a rational policy for their treatment. 23 of these patients were seen within the last 10 years and thirteen within the preceding 30 years. The follow-up periods range from 3 to $4^{\mathrm{I}}$ years.

\section{Clinical material}

PRESENTATION

In nineteen patients, the presence of a tumour in the anterior optic pathways was heralded by ophthalmic symptoms: visual loss in eight, proptosis in eight, strabismus in two, and nystagmus in one. In fifteen of these patients, the diagnosis was confirmed before the age of 5 years (average age- 3 years). In the other four, the tumour caused ocular symptoms that were not explained until the patients were 10, 20, 26, and 40 years old respectively.

Amblyopia and pallor of an optic disc discovered incidentally during a routine preschool examination led to the diagnosis in seven patients.

In nine children, from 7 months to 9 years old, the tumour was identified in the course of paediatric and radiological investigations for such non-ocular problems as plexiform facial neuroma, ataxia, mental retardation, diabetes insipidus, precocious puberty, neonatal jaundice, and hydrocephalus.

One glioma was found incidentally at autopsy in a 64-year-old woman. 
Optic glioma was verified histologically in 21 of the 36 patients. In six more, the tumour was identified by neuroradiological studies and direct observation at craniotomy. In the remaining nine (all children with neurofibromatosis, amblyopia, pale optic discs, and enlarged optic canals on one or both sides) the diagnosis was presumptive. Tomographic pneumoencephalography performed in four of this last group of nine children revealed an anteriorly situated mass in the third ventricle involving the chiasm and one or both optic nerves. Proptosis of the amblyopic eye provided strong evidence of optic glioma in three children. In the remaining two, sphenoidal dysplasia on the side of the dilated optic canal and a "J-shaped" sella supported the diagnosis.

\section{LOCATION AND EXTENT OF THE TUMOUR}

In seven patients, the tumour was confined to the optic nerve, and in three of these the posterior limit was intracranial. The tumours in 29 patients occupied the chiasm and the hypothalamic structures bordering the third ventricle. There was expansion of one or both optic nerves in all but two of these patients, while in six there was gross evidence of involvement of an optic tract at craniotomy.

The size and limits of the chiasmal tumours at the time of diagnosis could be defined only in general terms: very extensive, involving wide areas of adjacent brain (seven cases); intermediate (fifteen cases); and relatively small (seven cases). On subsequent radiography, there was no evidence that any tumour had changed in extent.

\section{ASSOGIATED DISORDERS}

\section{Neurofibromatosis}

Unequivocal signs occurred in sixteen patients and probable signs in five patients Neurofibromatosis was present in the families of seven patients with optic glioma. In two patients there was a family history of optic glioma, one occurring in a sibling, the other in a mother.

\section{Hypothalamic signs}

These were recorded in twelve patients, all of whom had a tumour of the chiasm and anterior third ventricle. Precocious puberty was present in eight patients, seven of whom had neurofibromatosis. Other disorders seen were girdle-type obesity, radiological evidence of increased bone age, very rapid growth at the onset of puberty, diabetes insipidus, panhypopituitarism, obesity with dwarfism, and obesity with hypersomnia.

\section{Hydrocephalus}

This was progressive and fatal in four patients with extensive intracranial tumours. Two infants were almost blind, had enlarging heads, and showed radiological signs of spreading cranial sutures and ventricular enlargement. Despite courses of radiation therapy, they died at 2 and 4 years of age. Two other patients developed signs of raised intracranial pressure at 5 and 6 years of age; both had generalized neurofibromatosis and severe visual loss. One was treated with irradiation and survived for so years. The other had two unsuccessful operations for hydrocephalus.

Another child with poor vision and signs of hydrocephalus was treated with a ventriculoatrial shunt and irradiation. She is alive and well 8 years after her operation. 
All of the above patients had severe bilateral visual loss in infancy or early childhood, ranging from blindness or near blindness in eight eyes to 20/70 acuity with temporal hemianopsia in two eyes.

\section{Benign asymmetrical ventricular dilatation}

This was observed to be unassociated with clinical signs of hydrocephalus in three children with chiasmal tumours. One received radiation therapy; the others did not. All are living and healthy.

\section{MORTALITY FROM OTHER CAUSES}

One 6-year-old boy died 12 hours after attempted resection of a glioma in the chiasm and optic nerves.

Three patients with inactive optic gliomata and generalized neurofibromatosis who were followed for 15 to 18 years died from sarcomatous degeneration in an extracerebral neurofibroma.

One elderly patient died after several strokes. She had an optic nerve and chiasmal glioma that had been diagnosed clinically and verified histologically $4 \mathrm{I}$ years before her death.

A 64-year-old woman, who died in a mental institution from pneumonia and Alzheimer's disease, had a chiasmal and optic nerve glioma that was first discovered at autopsy.

\section{VISUALI IMPAIRMENT}

The initial visual acuity of the 36 patients may be summarized as follows:

$\begin{array}{ll}20 / 200 \text { or less } & 29 \text { eyes } \\ 20 / 50 \text { to } 20 / 200 & \text { I I eyes } \\ 20 / 20 \text { to } 20 / 40 & 32 \text { eyes* }\end{array}$

The visual acuity in relation to untreated optic gliomata is summarized in Table I and that in eyes with irradiated optic gliomata in Table II (overleaf). Of the fourteen patients whose eyes were irradiated, three were not treated until late in the follow-up period when their vision had begun to fail.

PROPTOSIS

The course of proptosis in the eight patients who demonstrated it is shown in Table III (overleaf).

SURGICAL PROCEDURES

In seven patients the optic nerve was excised, one by the Krönlein approach and six by a transcranial approach. Only three of these eyes were blind. The tumour was totally excised in four of the seven patients and partially excised in three (the glioma being trans-sected intracranially in two of the last three and within the orbit in one). During the years that these last three patients were observed, none showed signs of visual loss in their remaining eye, although chiasmal tumour was known to be present.

\section{COMPLIGATIONS}

A total homonymous hemianopsia developed in one young patient after biopsy of the lateral aspect of her tumour. Now, 13 years later, the hemianopsia is her predominant visual handicap; the unresected tumour has caused no further loss of visual function. 
Table I Visual acuity in eyes with untreated tumours: 4 I eyes (23 patients)*

\begin{tabular}{|c|c|c|c|c|}
\hline \multirow{2}{*}{ Location } & \multirow{2}{*}{$\begin{array}{l}\text { No. of } \\
\text { eyes }\end{array}$} & \multicolumn{3}{|l|}{ Visual acuity } \\
\hline & & Deteriorated & Stable & Improved \\
\hline Optic nerve & 5 & 2 & 3 & - \\
\hline Optic nerve and chiasm & 18 & 2 & 14 & 2 \\
\hline Optic nerve, chiasm, and hypothalamus & 18 & 6 & 10 & 2 \\
\hline Total & 41 & Io & 27 & 4 \\
\hline
\end{tabular}

* Follow-up: $3-5$ yrs (4 patients); 5-10 yrs (I I patients); 10-20 yrs (5 patients); over 20 yrs (3 patients)

Table II Visual acuity in eyes with irradiated tumours: 28 eyes (I4 patients)*

\begin{tabular}{|c|c|c|c|c|}
\hline \multirow{2}{*}{ Location } & \multirow{2}{*}{$\begin{array}{l}\text { No. of } \\
\text { eyes }\end{array}$} & \multicolumn{3}{|l|}{ Visual acuity } \\
\hline & & Deteriorated & Stable & Improved \\
\hline Optic nerve and chiasm & 8 & I & 6 & I \\
\hline Optic nerve, chiasm, and hypothalamus & 20 & 5 & 12 & 3 \\
\hline Total & 28 & 6 & 18 & 4 \\
\hline
\end{tabular}

* Follow-up: Birth to 5 yrs (4 patients); $5^{-10}$ yrs (4 patients); ro-20 yrs (4 patients); over 20 yrs ( 2 patients)

Table III Course of proptosis: 8 eyes (1 2 patients)

\begin{tabular}{|c|c|c|c|c|c|}
\hline \multirow{2}{*}{ Treatment } & \multirow{2}{*}{ Site } & \multicolumn{4}{|l|}{ Proptosis } \\
\hline & & Increased & Constant & Decreased & Total \\
\hline \multirow[t]{4}{*}{ None } & Optic nerve & 2 & 2 & - & 4 \\
\hline & Optic nerve and chiasm & $\mathbf{I}$ & $\mathbf{I}$ & - & 2 \\
\hline & Optic nerve, chiasm, and hypothalamus & - & I & $\mathbf{I}$ & 2 \\
\hline & Total & 3 & 4 & $\mathbf{I}$ & 8 \\
\hline Irradiation & Optic nerve, chiasm, and hypothalamus & $\mathbf{I}$ & 2 & $\mathbf{I}$ & 4 \\
\hline
\end{tabular}

A variety of orbital complications resulted from efforts to excise as much of the optic nerve as possible. These included ocular ischemia, muscle palsies, and ptosis.

\section{Discussion}

The clinical features and course of optic gliomata during childhood and adolescence justify their inclusion in a special category among glial tumours of the brain. Our study suggests that they are congenital, non-neoplastic, self-limiting tumours. 
The theory of the congenital origin of optic gliomata was advocated by Hudson (I9I2) and Verhoeff (1922). Our report lends support to this theory because 80 per cent. of the tumours that we saw were diagnosed in infants and preschool children, and ocular signs were chronic when first seen.

The paucity of neurological symptoms in the young patient with an optic glioma is deceptive. Very few of these congenital tumours are confined exclusively to an optic nerve and even fewer to the orbit alone. The chiasm, one or both optic nerves, and adjacent portions of the brain were usually involved from the time of the initial diagnosis in our series, and in those reported by Martin and Cushing (1923), Jefferson (1940), Walsh (1956), and Tym ( 1961 ). In two-thirds of our patients, the ocular signs of this involvement appeared before school age. The systemic signs of hypothalamic disturbance appear later and are usually mild. Precocious puberty in these patients is evidence of intracranial tumour, but has no prognostic significance; the frequent association of this disorder with neurofibromatosis was stressed by Sigurjonsdottir and Hayles (1968) and was apparent in our patients. Diabetes insipidus, somnolence, and progressive mental deterioration signify an extensive tumour with a poor long-term prognosis. Progressive hydrocephalus is uncommon, and in infants is fatal within 2 to 3 years. When the onset of hydrocephalus is delayed, the child may survive until adolescence.

The long-term effects of optic gliomata on visual acuity in this series were of great significance. At the time the tumour was diagnosed, visual loss was already severe in 45 per cent. of the eyes. However, in 40 per cent. of involved eyes, vision was excellent or good. The ratio of these two groups did not change during follow-up periods of from io to 20 years.

Optic gliomata are clinically indolent tumours that tend to enlarge, cause symptoms early in life, and remain static thereafter. Unlike the rare glioblastomata that arise in the adult optic nerve (Saebø, I949; Mattson and Peterson, I966), childhood gliomata do not undergo malignant degeneration (Verhoeff, I922; del Rio-Hortega, I940; Davis, I968). They have features of congenital hamartomata.

Clinically and histologically, optic gliomata belong to a unique group of congenital glial tumours encountered in the cerebellum, medulla, corpus callosum, and spinal cord (del Rio Hortega, I940; Bucy and Thieman, 1968). The optic gliomata differ from the other members of this group primarily in their frequent concurrence with neurofibromatosis.

Optic gliomata expand without invasion of the surrounding tissue (Christensen and Ry Anderson, 1952). Two mechanisms account for their growth: collateral hyperplasia of adjacent glia and connective tissue (Verhoeff, 1922), and production of intra- and extracellular mucosubstance (Anderson and Spencer, 1970). The absence of mitoses is evidence that these tumours do not enlarge by cell division or invasion (Verhoeff, 1922). Cystic regrowth from the cut end of a tumour is exceedingly rare and is a non-neoplastic process (Wolter and McKenney, 1964).

\section{Management}

Knowledge of the natural history of optic gliomata, as documented in this study, provides the only rational basis for therapy.

Transcranial operations on the tumour do not prolong life; in fact, excision or manipulation of the chiasmal mass during biopsy may increase morbidity and mortality (Tym, 1961). Recurrent increases in intracranial pressure constitute the only indication for 
neurosurgical treatment. However, the shunting procedures employed often proved disappointing in our cases and in similar cases reported by others (Chutorian, Schwartz, Evans, and Carter, 1964).

Exploratory craniotomy is negated by modern neuroradiological procedures which permit accurate diagnosis and assessment of the extent of the tumour.

Irradiation of chiasmal gliomata has not influenced the natural history of those we have studied. Taveras, Mount, and Wood (1956) advocated radiotherapy, but their favourable visual responses may have coincided with spontaneous improvement as reported by Tym ( 1961 ) and as exemplified by this report.

Excision of the tumour is justified only for the relief of severe proptosis of a blind eye.

Incomplete excision is no sin, as Hudson (1912) established so convincingly. It can be accomplished through a lateral or medial orbitotomy, with preservation of ocular mobility and satisfactory external appearance as goals of the operation.

\section{Summary}

Prolonged follow-up of $3^{6}$ children with optic glioma confirms that the tumour is congenital, non-neoplastic, and self-limiting, and has a good prognosis for life.

Visual impairment, present at the time of diagnosis, does not change appréciably thereafter.

Excision of such a tumour should be undertaken only to control proptosis in a blind eye.

Life cannot be prolonged by irradiation or transcranial procedures on the tumour.

The authors wish to acknowledge their gratitude to Dr. J. E. K. Galbraith (Royal Melbourne Hospital) for his review and assistance with this manuscript.

\section{References}

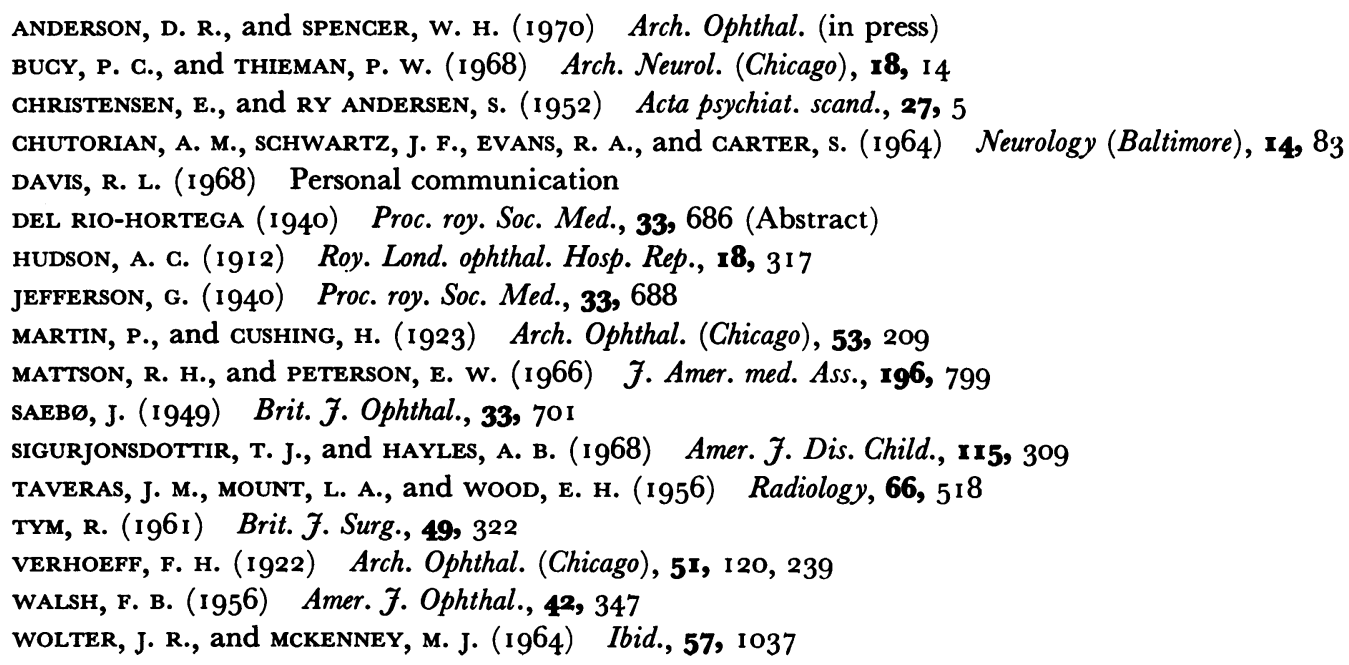

